\title{
Interpreting Offence in Advertising: A Regulatory Perspective: An Abstract
}

\author{
Kristina Auxtova and Mary Brennan
}

\begin{abstract}
Given the lack of attention paid to the interpretation of offensive advertising by different stakeholder groups and the actual offence caused, this study aims to explore how offence and harm have been attributed to non-profit advertising by complainants and regulators and how the associated organisations respond in light of these formal complaints and adjudications. We content analyse 9,055 consumer complaints received by the Advertising Standards Authority (ASA), UK, in the period of 2009-2015 as well as the associated 309 complaint adjudication reports concerning offensive and harmful advertising within the non-profit sector. Our findings suggest that charities (38\%) and issues related to children (46\%) are most often complained about. A thematic analysis of the rulings shows certain disconnects in the discourses across the three stakeholder groups (complainants, regulators, associated organisation) with the current self-regulatory system appearing to favour the industry. The understanding of these discourses helps to contribute to the ongoing debates on the appropriateness, ethics and application of offensive themes, formats and imagery in non-profit advertising as well as the debates on effectiveness of self-regulation.
\end{abstract}

This research is funded by the ESRC and the University of Edinburgh.

K. Auxtova $(\varangle) \cdot$ M. Brennan

University of Edinburgh, Edinburgh, UK

e-mail: kristina.auxtova@ed.ac.uk; mary.brennan@ed.ac.uk 